\title{
Global electromagnetic simulations of the outer core of an ASDEX Upgrade L-mode plasma
}

\author{
J. Abiteboul, ${ }^{1, \text { a) }}$ T. Görler, ${ }^{1}$ F. Jenko, ${ }^{1,2}$ D. Told,,${ }^{1,2}$ and the ASDEX Upgrade Team ${ }^{1}$ \\ 1) Max-Planck-Institut für Plasmaphysik, Boltzmannstr. 2, 85748 Garching, \\ Germany. \\ ${ }^{2)}$ Department of Physics and Astronomy, University of California, Los Angeles, \\ California 90095, USA.
}

The outer core of a low confinement discharge performed at the ASDEX Upgrade tokamak is investigated using both global and local nonlinear gyrokinetic simulations. Previous work [D. Told et al., Phys. Plasmas 20, 122312 (2013)] had shown that local gyrokinetic simulations agree reasonably well with experimental results in terms of transport levels, with minor discrepancies that can be resolved within the uncertainties of the experimental profile. In the present work, the analysis of the same discharge is extended to include global gradient-driven simulations with the GENE code, taking into account the plasma profiles from mid-radius up to close to the separatrix. It is shown that the mean fluxes obtained assuming the local approximation are in general agreement with results from global simulations. Moreover, both types of simulations exhibit large-scale avalanche-like events, both in the ion and electron heat fluxes, with similar basic properties. However, analyzing the statistics of the fluxes reveals that intermittency is more important in the global case. Furthermore, even when averaging over turbulent time scales, radial corrugations in the ion temperature profile are identified in the global simulation. These corrugations are at most of the order of $1 \%$, but correspond to up to $10 \%$ variations in the gradient. Thus, while the results on the mean fluxes support the validity of the local approximation, the presence of corrugated structures may pose a challenge for the direct validation of local gyrokinetic simulations against experiments.

\footnotetext{
a)Electronic mail: jeremie.abiteboul@gmail.com
} 


\section{INTRODUCTION}

An essential challenge for the production of energy by magnetical confinement fusion devices is the understanding and prediction of turbulent transport in the plasma. From a solid theoretical basis (see Ref. 1 for a review), modern gyrokinetic theory has been established as the adequate framework for the description of turbulent transport in hightemperature magnetically confined plasmas. Thus, a number of simulation codes have been developed to solve this 5-dimensional system of equations, encompassing different levels of physical scope and using a variety of numerical methods (see Ref. 2 for a review) In recent years, applications of these codes have moved from a mostly qualitative approach to actual validation of the model against experiments.

In particular, one such example of a validation effort is in the outer core region of low confinement (so-called L-mode) tokamak plasmas, where gyrokinetic simulations were initially unable to reproduce experimental observations in specific DIII-D discharges, reporting a systematic underprediction of the transport levels. ${ }^{3,4}$ A more extensive study, including benchmarks between several codes, has since demonstrated that this discrepancy is less than initially suspected, and can be removed using so-called "flux-matching" procedures, where the ion temperature gradient is varied within the error bars associated to the experimental measurements. ${ }^{5}$ Moreover, no significant underprediction was identified in investigations for similar discharges in the Alcator C-MOD, ${ }^{6} \mathrm{NSTX}^{7}$ and ASDEX Upgrade ${ }^{8}$ tokamaks. In addition to comparisons of transport level, an ongoing effort also attempts to make direct comparisons of more detailed measurements from the experiments, ${ }^{9-13}$ such as fluctuation levels, wavenumber spectra and cross-phases between the fluctuating quantities. Overall, these studies show satisfying agreement between experiments and simulations, when taking into account the large experimental error bars and the sensitivity of the codes to input parameters. This sensitivity is especially important with regards to the ion temperature gradient because of profile stiffness near the critical gradient, which implies that small uncertainties on the gradient can lead to substantial differences in transport levels in the simulations.

Nevertheless, a common limitation of these investigations is that they were performed using local gyrokinetic simulations (also referred to as flux-tube). This type of simulations makes use of the local approximation, assuming that the turbulent dynamics responsible for transport occur on small scales with respect to the characteristic scales of the 
background profiles and gradients. This allows for the use of periodic boundary conditions and pseudo-spectral methods, leading to a reduced numerical cost with respect to global simulations, which consider an extended radial simulation domain, taking into account the plasma profiles. Local simulations have previously been shown to agree well with global simulations in the local limit. ${ }^{14-18}$ However, this was mostly demonstrated in "ideal" simulation setups incorporating reduced physics models.

In this paper, we present an comparison of local and global gyrokinetic simulations under realistic experimental conditions. The test-case for this study is the outer core of an ASDEX L-mode discharge, previously analyzed with local gyrokinetic simulations. ${ }^{8}$ This discharge exhibits a stationary phase designed to reproduce the parameters of current ramp scenarios. ${ }^{19}$ Global simulations, including experimentally measured profiles and geometries, are performed from roughly mid-radius up to close to the separatix. Both ion and electron kinetic physics are included, as well as electromagnetic effects and collisions. In terms of mean fluxes, the results of local and global simulations show discrepancies, but these remain within the expected errors due to uncertainties in the measurement and to strong profile stiffness. Analysis of statistical properties of the turbulence show that large-scale avalanche-like events are observed in both cases, with more intermittency reported in the global simulation. Finally, time-independent structures are observed in the global case, with strong local variations of the temperature gradient. Such corrugations may present a challenge for the validation of gyrokinetic simulations.

This paper is organized as follows. In section II, the gyrokinetic code GENE used in the present study is described, with a specific emphasis on the difference between local and global simulations. Comparisons of mean transport levels between these two types of simulations are performed in section III. A detailed study of the statistical properties of the underlying turbulence is presented in section IV, including in particular the observation of corrugated structures in global simulations. Conclusions and a discussion of implications for future validation studies follow. 


\section{METHODOLOGY}

\section{A. Brief description of the simulation characteristics}

The simulations are performed using the Eulerian nonlinear gyrokinetic code GENE, ${ }^{20}$ which self-consistently solves the coupled Vlasov and Maxwell equations in either local or global simulation domains. A detailed presentation of the equations solved and their numerical implementation can be found in Ref.21, we focus here only on the specifics of the simulations considered.

First of all, both ions (deuterons) and electrons are treated gyrokinetically with realistic mass ratio, including both intra- and inter-species collisions. These are implemented in the form of a linearized Landau-Boltzmann collision operator, ${ }^{22}$ including both pitchangle and energy scattering, as well as additional terms ensuring conservation of density, momentum, and energy. Electromagnetic effects are also taken into account by solving for the parallel component of Ampère's law, the perpendicular component (i.e., parallel magnetic field fluctuations) being negligible due to the low plasma $\beta$ in the experiments. The geometry is taken directly from the experimental geqdsk file by the method of field line tracing. ${ }^{23}$ Thus, the metric coefficients used in the simulation are constructed to reflect the geometry of the studied discharge.

Note that, although electrons are treated gyrokinetically, the full range of scales is not considered as electron temperature gradient (ETG) driven turbulence has been found to play only a minor role in the specific experimental conditions considered here. ${ }^{8}$ Numerical dissipation is introduced in the parallel direction through a fourth order centered finite difference scheme, in order to damp grid-scale modes. ${ }^{24}$ Equilibrium rotation is not included in the simulations, as the experiments considered here exhibited both small rotation velocity and small rotation shear.

\section{B. Local vs global gyrokinetic simulations}

One of the defining aspects of turbulence in tokamak plasmas, as opposed for instance to fluid turbulence, is the presence of a background magnetic field, which leads to a strong anisotropy of the turbulence. More precisely, the low frequency perturbations we are interested in display very long wavelengths in the direction parallel to the magnetic 
field with respect to perpendicular wavelength, which remain of the order of the Larmor radius. This important geometric aspect is one of the key elements of the gyrokinetic ordering, ${ }^{1}$ but it can be further used to perform efficient numerical simulations. For instance, one can adopt so-called field-aligned coordinates, ${ }^{25}$ as is done in the gyrokinetic code GENE, which allows to gain roughly an order of magnitude in simulation costs.

A further approximation can be made by considering for the simulation only a narrow radial domain in the vicinity of a given flux-surface. This is the so-called local, or fluxtube, approximation. ${ }^{25}$ This amounts to an expansion of the system of equations around a given radial position, leading to a constant value of the profiles, gradients, geometric coefficients, etc. This approach is typically valid for the case $\rho_{*} \rightarrow 0$, where we define the normalized gyroradius as $\rho_{*}=\rho_{i} / a$ where $\rho_{i}$ is the thermal ion Larmor radius and $a$ is the minor radius of the tokamak. The advantage of adopting this approximation is that the domain becomes periodic, and pseudo-spectral methods can be employed, allowing for relatively cheap numerical simulations. Or, equivalently, to include more physics content for the same numerical cost. Naturally, the main drawback of such a modeling approach lies in the implicit scale separation between equilibrium and fluctuations. This means that a number of nonlocal effects, such as turbulence spreading from unstable to stable regions of the plasma, ${ }^{26,27}$ cannot be taken into account.

In order to tackle such issues, a number of global gyrokinetic codes have been developed $^{2}$ and are able to account for a wide radial simulation domain, at the expense of increased numerical costs. In order to test the validity of the local approximation, comparisons have been performed, ${ }^{14-16}$ basically proving that - at least for a specific set of observables - local simulations converge to the results of global simulations in the limit $\rho_{*} \rightarrow 0$, as expected from theory. However, these studies were performed for an "ideal" gyrokinetic test case, namely the so-called Cyclone base case. This set of parameters, although it has been very useful for the development and benchmark of gyrokinetic codes, presents a number of limitations. In particular, only ions are treated kinetically while the electron response is assumed adiabatic, and the geometry is taken as circular. Moreover, it corresponds to the inner core of a tokamak plasma, whereas global effects are expected to be more significant closer to the edge. Thus, although the previously cited comparisons can give us confidence in the efficiency of the local approximation, testing it in more realistic experimental conditions is of interest in order to assess the predictive capabilities of local gyrokinetic simulations. Recent studies have already begun to investigate this issue 
in the core of ASDEX Upgrade and JET plasmas. ${ }^{28}$

An important issue for the development and running of global gyrokinetic codes is that of the forcing. In local simulations, the mean background gradients provide a constant source of free energy, and no additional source is required. In global simulations however, in the absence of external forcing, the mean profiles would relax, killing the turbulence drive. Therefore an additional driving term is required, of which two main types can be identified. On the one hand, the most physically relevant forcing is to apply a given input source of energy, mimicking the heating by external sources in experiments. This is the so-called flux-driven boundary conditions, ${ }^{29}$ recently adapted to several gyrokinetic codes. ${ }^{16,30,31}$ The main drawback of this method is that, as the final profiles are not known a priori, simulations must be run over confinement time scales $\left(\propto \rho_{*}^{-3}\right)$ rather than turbulence time scales $\left(\propto \rho_{*}^{-1}\right)$, as is the case for local simulations. On the other hand, a more constrained way of running global simulations is to adopt the so-called gradient-driven method, which aims at keeping the mean gradients near their initial value in order to maintain a roughly constant turbulent drive. This is achieved by adding a Krook term to the right-hand side of the gyrokinetic equation, which basically maintains the distribution function near its initial equilibrium. ${ }^{21,32,33}$ In that sense, gradient-driven simulations assume certain properties of local simulations, namely a constant turbulent drive and a limitation to the system's exploration away from the initial equilibrium. Nevertheless, this allows one to perform global simulations, taking into account profile effects, on turbulent rather than confinement time scales. Moreover, setting a priori the mean profiles allows for more efficient comparisons to experimental results. Thus, this type of forcing will be adopted for global simulations in the present work, and one should keep in mind that, as in local simulations, the system may be somewhat over-constrained with respect to more realistic flux-driven simulations.

As mentioned, the profiles are maintained near their initial value in the global gradientdriven simulations through the implementation of Krook-type heat ${ }^{21}$ and particle ${ }^{34}$ sources in GENE. These sources, which conserve flux-surface averaged density and momentum, are applied over the whole radial simulation domain. The amplitude of the sources is set below the maximal linear growth rates, and varying them may in principle modify the degree of profile relaxation in the simulations. However, in the present case, varying them from 0.02 to $0.5 c_{s} / a$ does not significantly affect the results presented in the following. In order to allow for somewhat more self-organization of the system, the 
application of the Krook source is smoothed using Gaussian filters, both radially and in time. Additionally, to avoid spurious numerical instabilities, a separate Krook term of higher amplitude - comparable to the maximal linear growth rates - is added in a narrow domain near the radial boundaries. Radially, this additional term takes the form of a fourth-order polynomial, which is zero everywhere except in less than $10 \%$ of the radial domain near the boundaries.

Finally, it is interesting to compare the numerical costs of local and global simulations in this context. Well-resolved local simulations for the present ASDEX discharge require resolutions of 512 points in the radial direction, 48 modes in the binormal direction, and 32 points in the parallel spatial direction. The velocity space discretization requires $32 \times 20$ points in parallel velocity and magnetic moment. This corresponds to roughly $2.10^{9}$ grid points, since two species are treated kinetically. Interestingly, for a radial domain covering roughly half of the minor radius (see Fig. 1), global runs can be performed with similar spatial resolutions, with only, in some cases, a minor increase in the number of modes in the binormal direction. However, the variation of the temperature across the radial domain requires that a larger resolution be used in velocity space, with a doubling of the number of grid points both in parallel velocity and in magnetic moment. Most of the results presented here were obtained with the following grid: 512 radial points, 64 modes in the binormal direction, 32 points in the parallel direction, 64 points in parallel velocity, 20 points in magnetic moment. Thus, the overall increase of resolution going from local to global is typically a factor of 4 . However, the loss of periodicity in the radial direction means that simulations become more expensive. Indeed, the type of global simulations presented in this work require approximately 2 million core hours, roughly an order of magnitude more than the corresponding local simulations.

\section{COMPARISON OF TIME-AVERAGED FLUXES}

For this study, we focus on ASDEX Upgrade discharge \#28151, already well-documented in previous publications. ${ }^{8,19}$ This corresponds to an L-mode discharge, with a magnetic field of $2.3 \mathrm{~T}$ and a plasma current of $800 \mathrm{kA}$, where a stationary phase was established at physical parameters typical for a current ramp. For this phase in the discharge, the input power was $0.53 \mathrm{MW}$, from electron cyclotron resonance heating. The key plasma

profiles are given in Fig. 1, expressed in terms of logarithmic gradients with the following 


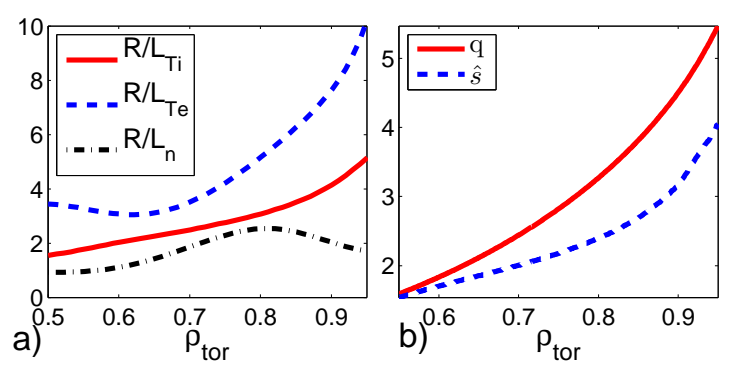

FIG. 1. (Color online) (a) Global profiles, as a function of the normalized toroidal magnetic flux, of the logarithmic gradients (see Eq.(1)) from discharge \#28151 for density, and ion and electron temperatures. (b) Global profiles of the safety factor and magnetic shear, defined as $\hat{s}=\left(\rho_{\text {tor }} / q\right)\left(d q / d \rho_{\text {tor }}\right)$.

definition for a given quantity $X$

$$
\omega_{X}=-\frac{1}{X} \frac{d X}{d \rho_{\mathrm{tor}}}
$$

where we choose as radial coordinate $\rho_{\text {tor }}=\sqrt{\Phi_{\text {tor }} / \Phi_{\text {edge }}}$ where $\Phi_{\text {tor }}$ is the toroidal magnetic flux and $\Phi_{\text {edge }}$ is its value at the last closed flux surface. We focus in the present work on the outer core of the plasma, from roughly mid-radius up to close to the separatrix. Considering the aim of the present work, it is also important to note that, in the radial domain considered, the normalized gyroradius $\rho_{*}$ in the experiment ranged from 1/300 near mid-radius to 1/900 approaching the separatrix. According to previous results in the Cyclone base case, ${ }^{14-16}$ this should correspond to a region where local and global results are in accordance. It has been suggested ${ }^{15}$ that the standard $\rho_{*}$ parameter is not the most adequate, as finite-size effects may occur for small values of $\rho_{*}$ when the actual driving region is narrower than the tokamak minor radius. However, the alternatives to $\rho_{*}$ identified in Ref. 15 assume a specific simulation setup, with a localized turbulent drive in the radial domain, using the width of this region to compute an effective $\rho_{*}$. Given the profiles considered in the present study, Fig. 1, this method for identifying the relevance of global effects cannot be directly applied here. Moreover, one should note that the gradients vary significantly in the domain, suggesting that global effects such as turbulence spreading could play a role.

The results are presented in terms of time and flux-surface averaged ion and electron heat fluxes in Fig. 2. The fluxes are normalized to so-called gyro-Bohm units, $c_{s} \rho_{s}^{2} P_{\text {ref }} / L_{\text {ref }}$ where $c_{s}, \rho_{s}$ and $P_{\text {ref }}$ are reference values of the ion sound speed, Larmor radius and pressure, taken at the center of the (global) simulation domain, and $L_{\text {ref }}$ is a a macroscopic 


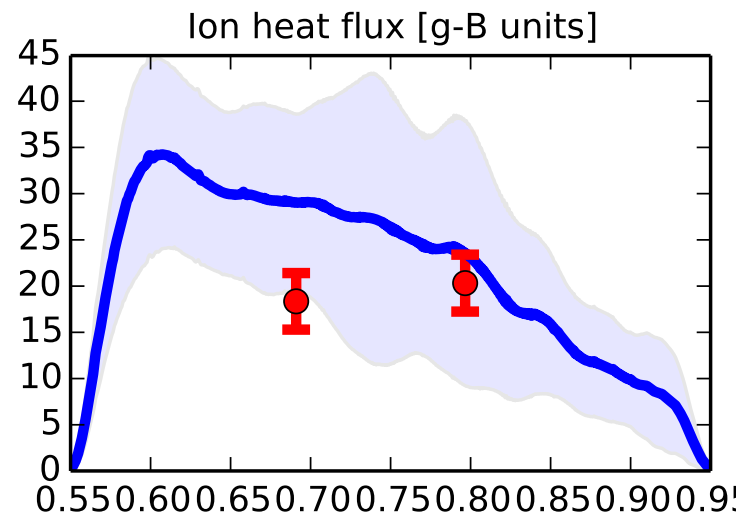

a) $\rho_{\text {tor }}$

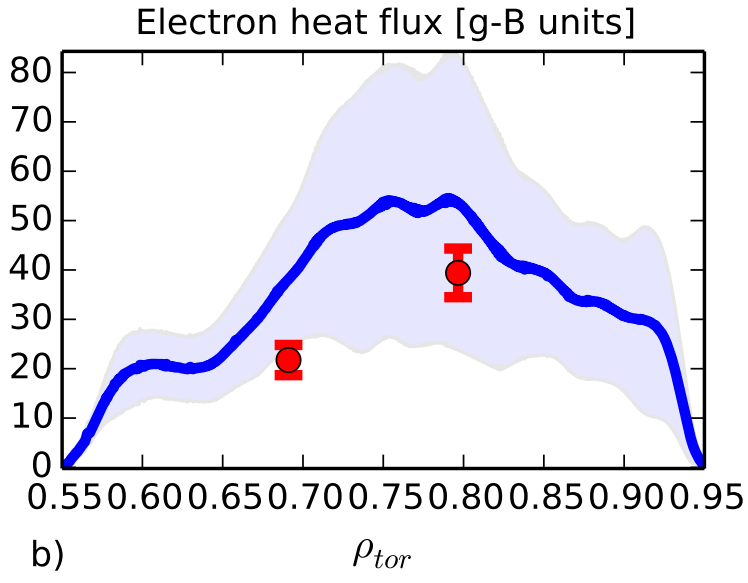

b)

FIG. 2. (Color online) Radial profile of the flux-surface and time averaged radial heat flux for (a) ions and (b) electrons, and comparison with local simulations. The error bars for the local runs (in red) indicate the uncertainty in the mean value due to intermittency, while the shaded (blue) area correspond to the standard deviation around the mean value in the global case.

reference scale which resembles a flux-surface averaged minor radius. ${ }^{8}$ The fluxes are timeaveraged over roughly $150 a / c_{s}$, after the simulation has reached a statistical steady-state. For the local simulations, the parameters are those of the "nominal" simulations from Ref. 8, i.e. directly using the parameters as measured in the experiments rather than adjusting them to match the observed heat fluxes, and without treating impurities as a separate kinetic species. The general observation is a reasonable agreement between the local and global simulations for both ion and electron heat fluxes, although the fluxes seem to be generally higher in the global case. In order to compare the results directly to experiments, it has been shown in the present experimental conditions ${ }^{8}$ that additional physics may need to be taken into account, especially with regards to the treatment of impurities. We focus instead on direct comparison between the local and global simulations, as their physics content is equivalent. Taking into account the profile stiffness identified in local studies of the same discharge, ${ }^{8}$ the minor discrepancies between local simulations and both global runs and experimental observations can all be resolved by modifying the driving gradients within the uncertainties of the experimental profile. Although reasonable agreement is obtained, one should not conclude that this is a direct validation, either of the gyrokinetic model as compared to experiments, or of the local approximation. Indeed, more stringent tests should be performed, such as exhaustive comparisons to experimental measurements, including more complex observables than mean heat fluxes. ${ }^{13}$ 

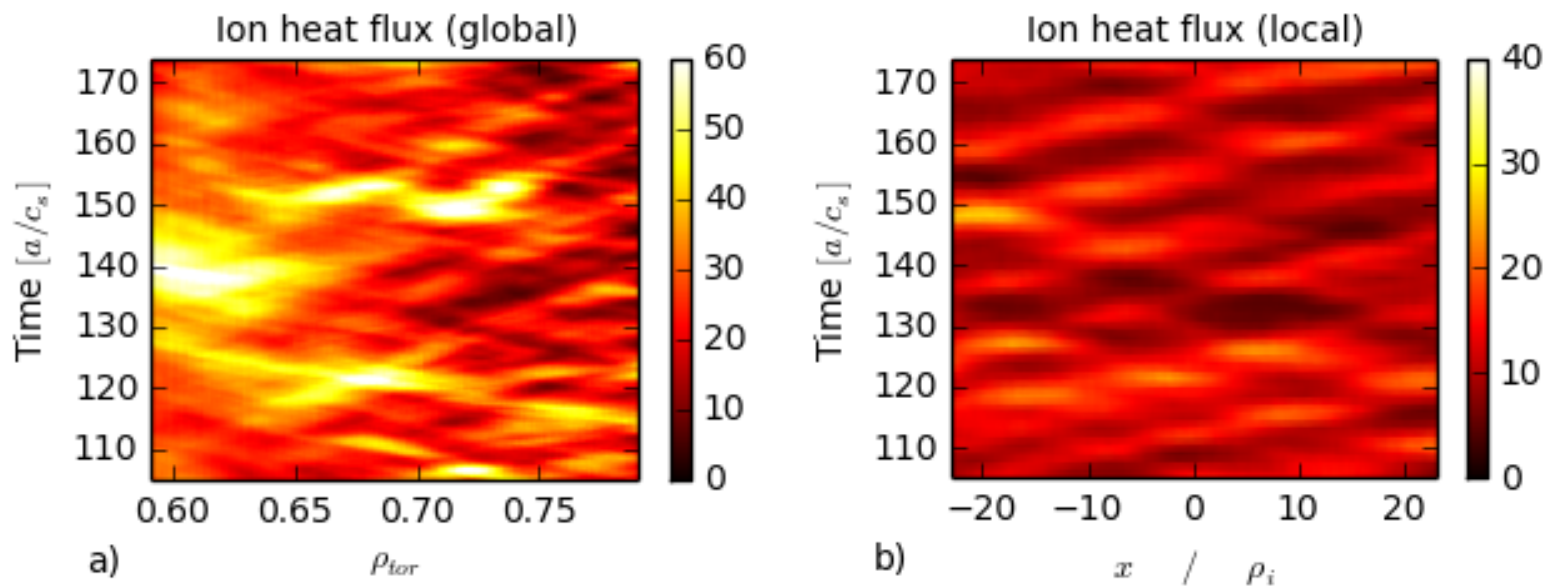

FIG. 3. (Color online) Two-dimensional (in radius and time) representation of the flux-surface averaged ion heat flux around $\rho_{\text {tor }}=0.691$, for (a) global and (b) local simulations. The fluxes are in reference gyro-Bohm units, taken at the center of the (global) simulation domain

Likewise, going beyond mean values of the fluxes, in the following section the statistical properties of turbulence in local and global simulations are analyzed in more detail.

\section{STATISTICAL ANALYSIS OF TURBULENT TRANSPORT}

\section{A. Heat flux avalanches}

In order to more precisely compare global and local gyrokinetic simulations, one can go beyond the mean value and investigate the dynamics of the turbulence, and in particular of the radial heat flux. We stress that, although it has been highlighted elsewhere ${ }^{35}$ that gyrokinetic turbulence does exhibit three-dimensional structures, as well as structures in velocity space, we focus here only on flux-surface averaged quantities. It should also be noted that although only the heat flux is considered here, other turbulent fluxes such as the toroidal momentum flux have been shown to robustly follow the dynamics of the heat flux..$^{36,37}$

The ion heat flux for both cases is displayed in Fig. 3, for the first radial position of the local simulations previously shown (i.e., $\rho_{\text {tor }}=0.691$ ). Namely, Fig. 3a corresponds to a zoom around this radial position for the global simulation, while Fig. 3b shows a symmetric domain around the center of the simulation of the same radial width. The radial transport clearly exhibits large-scale avalanche-like events. Comparing the detailed 

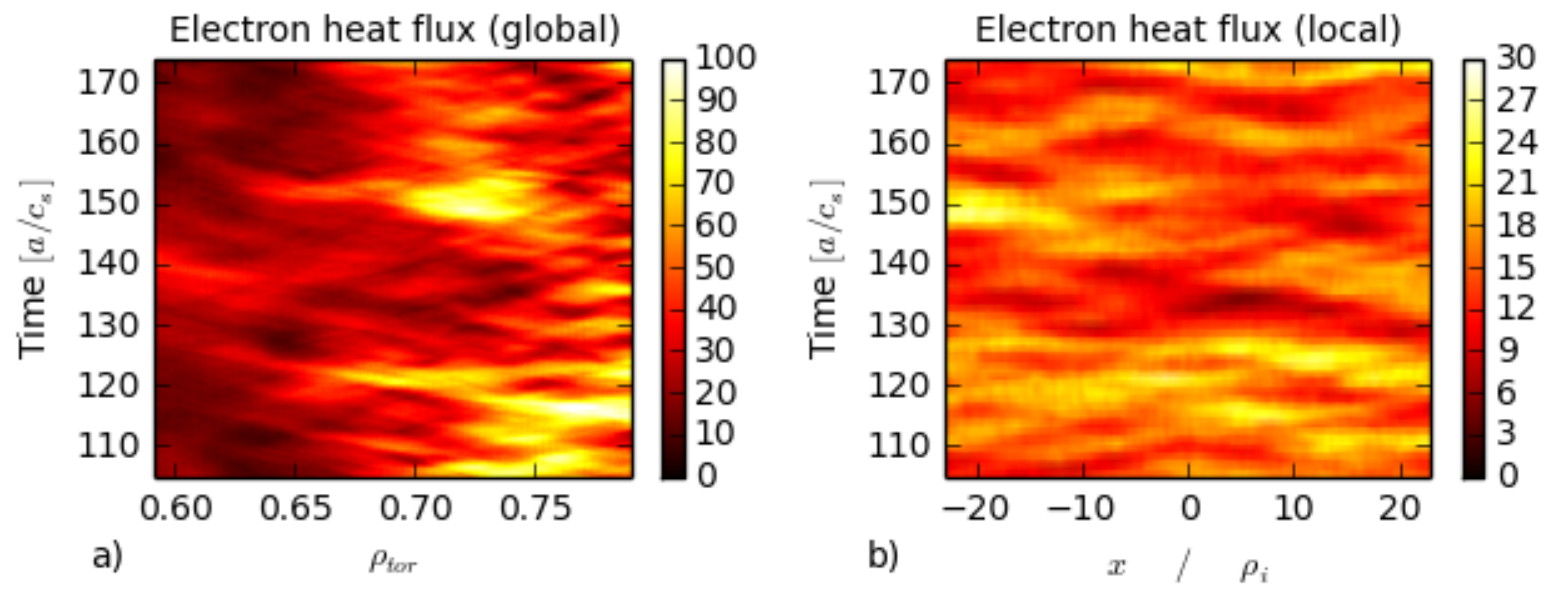

FIG. 4. (Color online) Two-dimensional (in radius and time) representation of the flux-surface averaged electron heat flux around $\rho_{\text {tor }}=0.691$, for (a) global and (b) local simulations. The fluxes are in reference gyro-Bohm units, taken at the center of the (global) simulation domain

properties of these avalanches, i.e. their velocity and radial extent, one finds no significant discrepancy between local and global fluxes, as previously reported in adiabatic electron simulations. ${ }^{16}$ One however notices that the relative amplitude of the heat flux maxima, which correspond to avalanches, with respect to the mean flux, appear stronger in the result from the global simulations, suggesting a higher level of intermittency.

Equivalent results are obtained when studying the electron fluxes, Fig. 4. Again the avalanches have similar properties in local and global simulations, with generally stronger peaks in the global case.

\section{B. Probability distribution functions of the fluxes}

In order to quantify these observations, one can analyze the turbulence statistics. In Fig. 5, the probability distribution functions (PDFs) of ion and electron heat flux are given, for $\rho_{\text {tor }}=0.691$. In both cases, data is taken only in the time domain after the system is considered to have reached a statistical steady-state. This corresponds to several hundred $a / c_{s}$. In the local simulation, all the points in the (periodic) radial direction are identical and can be used for the statistics, while for the global case we restrict the domain to \pm 0.05 around the reference position in $\rho_{\text {tor }}$. This is sufficient to obtain at least $5 \cdot 10^{5}$ data points in both cases. The general characteristics of the resulting PDFs, as well as those of the avalanches presented in the previous section, are comparable to previous 


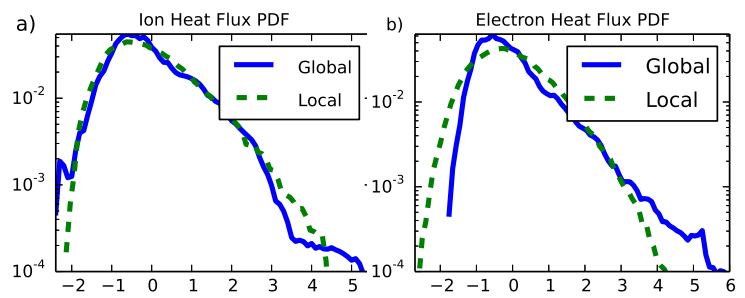

FIG. 5. (Color online) Normalized probability distribution functions (PDFs) from local and global simulations for (a) ion heat flux, and (b) electron heat flux. The x-axis corresponds to the fluxes normalized to their respective mean values and standard deviations.

detailed studies of turbulence properties (see for instance Ref. 36).

Visually, one can observe similar PDFs in the case of the ion heat flux, with possibly a larger tail in the global case, while for the electron heat flux the PDF obtained from the global simulation is more positively skewed and clearly shows a heavier tail. This can be investigated more precisely by quantifying the third and fourth order moments of the distribution functions. The third order moment, or skewness, defined as $S k(f)=\left\langle(f-\bar{f})^{3}\right\rangle /\left\langle(f-\bar{f})^{2}\right\rangle^{3 / 2}$ where $\bar{f}$ is the mean of $f$, is a measure of the asymmetry of a distribution. Here, in the ion case we find skewnesses of approximately 0.9 in the local case and 1.2 in the global case, the difference between the two being only marginally larger than the expected error bar. The disparity is more significant in the electron flux, with a skewness of 0.6 for the local simulation as compared to 1.9 in the global case, indicating positively skewed distributions in both cases with a much stronger asymmetry for the global flux. The fourth order moment, or kurtosis, defined as $K u(f)=\left\langle(f-\bar{f})^{4}\right\rangle /\left\langle(f-\bar{f})^{2}\right\rangle^{2}$, measures the weight of the heavy tails in the distribution. Considering here PDFs of heat fluxes, this is thus a measure of the importance in the distribution of high values of the flux due to avalanches. One finds here a significant deviation between local and global fluxes. In the global simulation, the kurtoses are of approximately 2.9 for ions and 6.5 for electrons, as compared to respectively 0.9 and 0.4 in the local case. This confirms the fact that intermittent transport is more prevalent in the global simulation. 


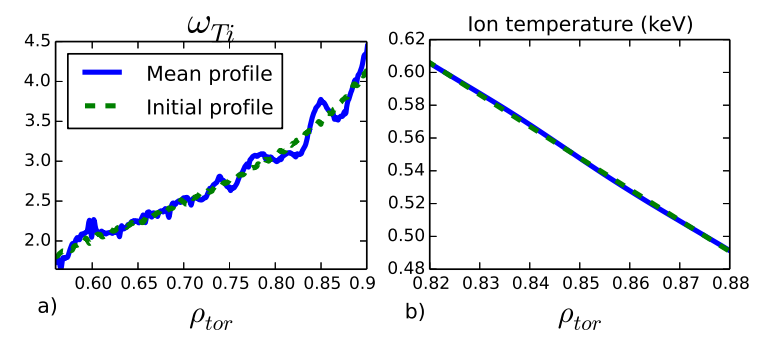

FIG. 6. (Color online) Radial profile of (a) logarithmic ion temperature gradient, and (b) ion temperature. The dashed green line corresponds to the target (initial) profile while the solid blue line is the final time-averaged profile

\section{Radial corrugations of the ion temperature gradient}

In global gradient-driven simulations, although the boundary conditions limit the system's excursion away from its initial state, they do not enforce that the mean gradients remain constant, and one can consider the dynamical evolution of the radial profiles. Indeed, one finds that structures in space may develop and endure in time. Fig. 6a shows the radial profile of the logarithmic ion temperature gradient, averaged over roughly $100 a / c_{s}$ (where $a$ is the minor radius and $c_{s}$ is the ion sound speed), i.e. much longer than a characteristic turbulent time scale. One observes strongly corrugated radial structures in the radial direction, of the order of $10 \%$ of the mean value. Note that these corrugations, although they may move radially, persist over even longer time scales, up to the duration of the longest simulations. On the one hand, such deviations in the gradient will only lead to small variations in the actual profile. This can be observed in Fig. 6b, which displays the ion temperature profile, for the same time average and with a radial zoom: the variation from the mean remains small, at most of the order of $1 \%$. Nevertheless, taking into account the strong profile stiffness often found in such experimental parameter domains, such a deviation in the driving gradient would correspond to a strong variation in the expected fluxes from local simulations. Thus, the presence of such corrugations may pose a difficult problem for the direct validation of local gyrokinetic simulations to experimental observations.

The exact origin of these corrugations remains to be fully understood. One could naturally draw a parallel to radial corrugations previously observed in gyrokinetic simulations and directly related to the physics of non-adiabatic passing electrons at low order mode rational surfaces. ${ }^{38,39}$ However, the structures observed here are clearly not tied 
to rational magnetic flux surfaces. Indeed, they do not appear to be fixed to an exact position and may move around slightly over time. Thus, they are perhaps more reminiscent of the self-organized structures identified in global flux-driven simulations and referred to as "staircases", ${ }^{40,41}$ in analogy with previous work from the geophysics community. However, since the more constrained gradient-driven simulations employed limit the complete self-organization of the system, and since the typical observations of "staircase" structures were for adiabatic electron simulations only, a one-to-one identification is not trivial. Long-lived zonal flow patterns have also previously been observed in both local and global gradient-driven simulations assuming adiabatic electron response. ${ }^{33}$ This is expected to be the subject of further investigation in the future.

\section{DISCUSSION AND CONCLUSIONS}

In this paper, we have presented global and local nonlinear gyrokinetic simulations of turbulence in the outer region of ASDEX Upgrade L-mode plasmas, using the GENE code. This is an important step for the validation of gyrokinetics against experiments, which has previously been undertaken employing mostly local simulations, with notable exceptions in recent years. ${ }^{17,18,28}$ Performing global comparisons is of course more challenging, in part because "flux-matching" procedures cannot be easily implemented, since global simulations consider as inputs global profiles rather than isolated values of the driving gradients. Nevertheless, systematic comparisons between global nonlinear simulations and experimental results could provide a more stringent validation test of gyrokinetics.

In terms of mean ion and electron heat fluxes, the local approximation seems to perform reasonably well with respect to global simulations. The agreement obtained is not perfect, but when taking into account the effect of profile stiffness it is expected to remain well within the expected uncertainty due to experimental error bars. Although this does not provide a direct validation of the local approximation, it is a good indication of its efficiency in such plasma parameters. A further level of comparison is to analyze the dynamics in time and in radius of the turbulent fluxes. Both for ions and electrons, one finds that the heat flux exhibits large-scale avalanche-like structures, with similar properties in both local and global simulations, albeit with stronger peaking in the global case. This is confirmed and quantified by an analysis of the probability distribution functions (PDFs) of the fluxes. These reveal similar structures but a higher kurtosis, 
indicating increased intermittency, in the global case, especially in terms of electron heat flux.

Finally, radial corrugations of the ion temperature gradient profile are found to persist over times much longer than typical turbulent time scales. These corrugations, which are not tied to mode rational surfaces, lead to variations of approximately $10 \%$ in terms of logarithmic gradients, which is significant in terms of turbulent drive, but only corresponds to variations of the order of $1 \%$ in the temperature profile, meaning that they should be challenging to observe in experiments. Indeed, due to the available data, experimental observations must assume some smoothness in the reconstructed profile, and may therefore not be able to observe such corrugated structures. The possible presence of such structures in experiments would pose difficult problems for the process of experimentally validating local gyrokinetic simulations.

As a conclusion, we stress that the global simulations presented here were performed with gradient-driven boundary conditions. This is an efficient solution for performing comparisons with experiments, as one is certain to remain near the experimental profiles, but it in principle limits the system's self-organization and excursion away from the initial state. In other words, though the Krook operator used to perform gradient-driven simulations does not prevent the emergence of self-organized structures such as the corrugations presented here, it does not allow for their fully self-consistent development. Thus, a more complete study of the radial corrugations observed here would need to be performed using flux-driven boundary conditions, where only the input flux is imposed and the system is allowed to evolve freely. Such simulations, which need to be run over confinement time scales with kinetic ions and electrons, are not presently accessible with the available codes and resources. However, in the near future, they could provide the most complete validation study for gyrokinetics.

\section{ACKNOWLEDGMENTS}

The authors acknowledge useful discussions with A. Bañon Navarro, G. Dif-Pradalier and H. Doerk, as well as fruitful interactions with participants at the "Festival de Théorie" held in Aix-en-Provence in 2013. The computations for the present study were carried out in part on the Helios computer of the IFERC-CSC. The research leading to these results has received funding from the European Research Council under the European Unions 
Seventh Framework Programme (FP7/2007-2013)/ERC Grant Agreement No. 277870.

\section{REFERENCES}

${ }^{1}$ A. J. Brizard and T. S. Hahm, Rev. Mod. Phys. 79, 421 (2007).

${ }^{2}$ X. Garbet, Y. Idomura, L. Villard, and T.-H. Watanabe, Nucl. Fusion 50, 043002 (2010).

${ }^{3}$ C. Holland, A. E. White, G. R. McKee, M. W. Shafer, J. Candy, R. E. Waltz, L. Schmitz, and G. R. Tynan, Phys. Plasmas 16, 052301 (2009).

${ }^{4}$ T. L. Rhodes, C. Holland, S. P. Smith, A. E. White, K. H. Burrell, J. Candy, J. C. DeBoo, E. J. Doyle, J. C. Hillesheim, J. E. Kinsey, et al., Nucl. Fusion 51, 063022 (2011).

${ }^{5}$ T. Görler, A. E. White, D. Told, F. Jenko, C. Holland, and T. L. Rhodes, Phys. Plasmas 21, 122307 (2014).

${ }^{6}$ N. T. Howard, A. E. White, M. Greenwald, M. L. Reinke, J. Walk, C. Holland, J. Candy, and T. Görler, Phys. Plasmas 20, 032510 (2013).

${ }^{7}$ Y. Ren, W. Guttenfelder, S. M. Kaye, E. Mazzucato, R. E. Bell, A. Diallo, C. W. Domier, B. P. LeBlanc, K. C. Lee, M. Podesta, et al., Nucl. Fusion 53, 083007 (2013). ${ }^{8}$ D. Told, F. Jenko, T. Görler, F. Casson, E. Fable, and ASDEX Upgrade Team, Phys. Plasmas 20, 122312 (2013).

${ }^{9}$ A. Casati, T. Gerbaud, P. Hennequin, C. Bourdelle, J. Candy, F. Clairet, X. Garbet, V. Grandgirard, Ö. Gürcan, S. Heuraux, et al., Phys. Rev. Lett. 102, 165005 (2009).

${ }^{10}$ A. E. White, L. Schmitz, W. A. Peebles, T. L. Rhodes, T. A. Carter, G. R. McKee, M. W. Shafer, G. M. Staebler, K. H. Burrell, J. C. DeBoo, et al., Phys. Plasmas 17, 020701 (2010).

${ }^{11}$ A. E. White, N. T. Howard, M. Greenwald, M. L. Reinke, C. Sung, S. Baek, M. Barnes, J. Candy, A. Dominguez, D. Ernst, et al., Phys. Plasmas 20, 056106 (2013).

${ }^{12}$ T. Happel, A. Banon Navarro, G. D. Conway, C. Angioni, M. Bernert, M. Dunne, E. Fable, B. Geiger, T. Görler, F. Jenko, et al., Phys. Plasmas 22, 032503 (2015).

${ }^{13}$ A. Banon Navarro, T. Happel, T. Görler, F. Jenko, J. Abiteboul, A. Bustos, H. Doerk, D. Told, and ASDEX Upgrade Team, Phys. Plasmas 22, 042513 (2015).

${ }^{14}$ J. Candy, R. E. Waltz, and W. Dorland, Phys. Plasmas 11, L25 (2004).

${ }^{15}$ B. F. McMillan, X. Lapillonne, S. Brunner, L. Villard, S. Jolliet, A. Bottino, T. Görler, 
and F. Jenko, Phys. Rev. Lett. 105, 155001 (2010).

${ }^{16}$ T. Görler, X. Lapillonne, S. Brunner, T. Dannert, F. Jenko, S. K. Aghdam, P. Marcus, B. F. McMillan, F. Merz, O. Sauter, D. Told, and L. Villard, Phys. Plasmas 18, 056103 (2011).

${ }^{17}$ C. Holland, J. C. DeBoo, T. L. Rhodes, L. Schmitz, J. C. Hillesheim, G. Wang, A. E. White, M. E. Austin, E. Doyle, W. A. Peebles, et al., Nucl. Fusion 52, 063028 (2012).

${ }^{18}$ C. Holland, C. C. Petty, L. Schmitz, K. H. Burrell, G. R. McKee, T. L. Rhodes, and J. Candy, Nucl. Fusion 52, 114007 (2012).

${ }^{19}$ E. Fable, C. Angioni, F. J. Casson, D. Told, F. Jenko, R. M. McDermott, F. Ryter, W. Treutterer, E. Viezzer, A. A. Ivanov, et al., Plasma Phys. Control. Fusion 55, 124028 (2013).

${ }^{20}$ F. Jenko, W. Dorland, M. Kotschenreuther, and B. N. Rogers, Phys. Plasmas 7, 1904 (2000).

${ }^{21}$ T. Görler, X. Lapillonne, S. Brunner, T. Dannert, F. Jenko, F. Merz, and D. Told, J. Comput. Phys. 230, 7053 (2011).

${ }^{22}$ F. Merz, Ph.D. thesis, Westfälische Wilhelms-Universität Münster (2008).

${ }^{23}$ P. Xanthopoulos and F. Jenko, Phys. Plasmas 13, 092301 (2006).

${ }^{24}$ M. J. Pueschel, T. Dannert, and F. Jenko, Comput. Phys. Commun. 181, 1428 (2010).

${ }^{25}$ M. A. Beer, S. C. Cowley, and G. W. Hammett, Phys. Plasmas 2, 2687 (1995).

${ }^{26}$ X. Garbet, L. Laurent, A. Samain, and J. Chinardet, Nucl. Fusion 34, 963 (1994).

${ }^{27}$ T. S. Hahm, P. H. Diamond, Z. Lin, K. Itoh, and S. I. Itoh, Plasma Phys. Control. Fusion 46, A323 (2004).

${ }^{28}$ F. Jenko, D. Told, T. Görler, J. Citrin, A. Navarro, Bañón Navarro, C. Bourdelle, S. Brunner, G. Conway, T. Dannert, H. Doerk, et al., Nucl. Fusion 53, 073003 (2013).

${ }^{29}$ X. Garbet, Y. Sarazin, P. Beyer, P. Ghendrih, R. E. Waltz, M. Ottaviani, and S. Benkadda, Nucl. Fusion 39, 2063 (1999).

${ }^{30}$ Y. Sarazin, V. Grandgirard, J. Abiteboul, S. Allfrey, X. Garbet, P. Ghendrih, G. Latu, A. Strugarek, and G. Dif-Pradalier, Nucl. Fusion 50, 054004 (2010).

${ }^{31}$ Y. Idomura, H. Urano, N. Aiba, and S. Tokuda, Nucl. Fusion 49, 065029 (2009).

${ }^{32}$ B. F. McMillan, S. Jolliet, T. M. Tran, L. Villard, A. Bottino, and P. Angelino, Phys. Plasmas 15, 052308 (2008).

${ }^{33}$ X. Lapillonne, B. F. McMillan, T. Görler, S. Brunner, T. Dannert, F. Jenko, F. Merz, and L. Villard, Phys. Plasmas 17, 112321 (2010). 
${ }^{34}$ D. Told, Ph.D. thesis, Universität Ulm. Fakultät für Naturwissenschaften (2012).

${ }^{35}$ P. Ghendrih, C. Norscini, T. Cartier-Michaud, G. Dif-Pradalier, J. Abiteboul, Y. Dong, X. Garbet, O. Gürcan, P. Hennequin, V. Grandgirard, et al., Eur. Phys. J. D 68, 303 (2014).

${ }^{36} \mathrm{~S} . \mathrm{Ku}$, J. Abiteboul, P. H. Diamond, G. Dif-Pradalier, J. M. Kwon, Y. Sarazin, T. S. Hahm, X. Garbet, C. S. Chang, G. Latu, E. S. Yoon, P. Ghendrih, S. Yi, A. Strugarek, W. Solomon, and V. Grandgirard, Nucl. Fusion 52, 063013 (2012).

${ }^{37}$ J. Abiteboul, P. Ghendrih, V. Grandgirard, T. Cartier-Michaud, G. Dif-Pradalier, X. Garbet, G. Latu, C. Passeron, Y. Sarazin, A. Strugarek, O. Thomine, and D. Zarzoso, Plasma Phys. Control. Fusion 55, 074001 (2013).

${ }^{38}$ R. E. Waltz, M. E. Austin, K. H. Burrell, and J. Candy, Phys. Plasmas 13, 52301 (2006).

${ }^{39}$ J. Dominski, S. Brunner, S. K. Aghdam, T. Görler, F. Jenko, and D. Told, J. Phys. Conf. Series 401, 012006 (2012).

${ }^{40}$ G. Dif-Pradalier, P. H. Diamond, V. Grandgirard, Y. Sarazin, J. Abiteboul, X. Garbet, P. Ghendrih, A. Strugarek, S. Ku, and C. S. Chang, Phys. Rev. E 82, 025401 (2010).

${ }^{41}$ G. Dif-Pradalier, G. Hornung, P. Ghendrih, Y. Sarazin, F. Clairet, L. Vermare, P. H. Diamond, J. Abiteboul, T. Cartier-Michaud, C. Ehrlacher, et al., Phys. Rev. Lett. 114, 085004 (2015). 